\title{
ACONSELHAMENTO CONTRACETIVO NA INTERRUPÇÃO VOLUNTÁRIA DE GRAVIDEZ: REVISÃO SISTEMÁTICA
}

\author{
Sara Palma ${ }^{1,2}$, Adriana Taborda $^{3}$, Nídia Nunes ${ }^{3}$, Mário Cardoso² e Maria Helena Presado $^{2}$ \\ ${ }^{1}$ Hospital Garcia de Orta, Almada, Portugal. saraelisabetepalma@gmail.com \\ ${ }^{2}$ Escola Superior de Enfermagem de Lisboa, Portugal. saraelisabetepalma@gmail.com; mmcardoso@esel.pt; \\ mhpresado@esel.pt \\ ${ }^{3}$ Hospital S. Bernardo, Setúbal, Portugal. alenurse.adriana@gmail.com; nidianunes@gmail.com
}

\begin{abstract}
Resumo. Em 2018 realizaram-se 14928 interrupções voluntárias de gravidez (IVG) em Portugal, $95,8 \%$ por opção da mulher. A principal causa invocada foi a toma incorreta e a descontinuidade do método contracetivo. A evidência científica sugere que a altura ideal para aderir a um contracetivo é durante a experiência vivida da IVG. Objetivos: Conhecer o impacto do aconselhamento contracetivo na escolha dos contracetivos pelas mulheres em processo de IVG. Método: Revisão da literatura realizada de 1 a 31 de janeiro de 2020, segundo as orientações do Joanna Briggs Institute e a questão "Qual o impacto do aconselhamento contracetivo realizado às mulheres em processo de IVG na escolha do método?". Recorreu-se à plataforma EBSCO e bases de dados CINAHL, MedicLatina e Cochrane Central Register of Controlled Trials, incluíram-se estudos em português, inglês ou espanhol em mulheres com idade igual ou superior a 16 anos. Resultados: Elegeram-se 11 estudos, entre 2014 e 2020. Todos na língua inglesa e nenhum realizado em Portugal. $\mathrm{O}$ aconselhamento favorece a escolha e a adesão aos métodos contracetivos, sendo influenciada pela qualificação dos profissionais, acessibilidade e disponibilidade gratuita de contracetivos. A análise qualitativa dos estudos, permitiram uma melhor compreensão dos aspetos psicossociais das mulheres em processo de IVG.
\end{abstract}

Palavras-Chave: Aconselhamento contracetivo; Métodos Contracetivos; Interrupção Voluntária da Gravidez.

\section{CONTRACEPTIVE COUNSELING ON VOLUNTARY TERMINATION OF PREGNANCY: SYSTEMATIC REVIEW}

Abstract. In 2018, there were 14928 voluntary termination of pregnancy's (VTP)in Portugal, 95.8\% at the option of women. The main reported cause was the incorrect use and discontinuity of the contraceptive. The scientific evidence suggest that the ideal time to adhere to a contraceptive is during the experiencing of the VTP. Goals: To know the impact of contraceptive counselling in the choice of contraceptive by women living VTP. Method: Literature review was carried out from 1 to 31 January 2020, following the guidelines of the Joanna Briggs Institute and the question "What contraceptive counselling is performed for women in the live VTP period and what is the impact on the choice of method?". EBSCO platform and CINAHL, MedicLatina and Cochrane Central Register of Controlled Trials databases were used, studies in Portuguese, English or Spanish in women 16 years old were included. Results: 11 studies were chosen, between 2014 and 2020. All in English and none developed in Portugal. Conclusion: Counselling favours the choice and adherence to contraceptive methods, being influenced by the qualification of professionals, accessibility and free availability of contraceptives. The qualitative analysis of the studies, allowed a better understanding of the psychosocial aspects of women in the process of VTP.

Keywords: Contraceptive Counseling; Contraceptive Methods; Voluntary Termination of Pregnancy. 


\section{INTRODUÇÃO}

Em Portugal é fácil o acesso à informação sobre contraceção e saúde sexual e reprodutiva. No entanto, isso não é sinónimo de fiabilidade, de utilização correta ou mesmo de uso de um método contracetivo (Águas, Bombas, \& Silva, 2016). Independentemente das razões apontadas para uma Interrupção Voluntária da Gravidez na base do problema encontramos a falha do método, o uso incorreto e a não adesão à contraceção (Palma \& Presado, 2019). As gravidezes não desejadas ameaçam a vida das mulheres com consequências na sua saúde e na oportunidade de terem uma vida economicamente mais favorável (Greene, Joshi, \& Robles, 2012).

Em Portugal, a Interrupção Voluntária da Gravidez (IVG) foi aprovada em 2007 com a Lei n6/84 de 11 de Maio, revogada pela Lei no16/2007 de 17 de Abril, no sentido da despenalização quando realizada por opção da mulher nas primeiras 10 semanas completas de gravidez.

A IVG processa-se em três momentos: o primeiro corresponde à consulta prévia onde é realizada a comprovação médica de que a gravidez não excede as 10 semanas, seguido de um período de reflexão, num tempo mínimo de três dias e após o qual, é recolhido o consentimento livre e esclarecido da grávida ou do representante legal para a realização do procedimento; o segundo momento corresponde à intervenção cirúrgica ou medicamentosa que termina efetivamente a gravidez; o último momento consiste na consulta de planeamento familiar realizada até 15 dias após a IVG e na prescrição de um método contracetivo (Lei 16/2007).

O aborto é um indicador de saúde pública que reflete não só a fertilidade da população, mas também a acessibilidade à contraceção. Ainda que a taxa de abortos em Portugal seja inferior à média europeia (DGS, 2019), verifica-se que a acessibilidade aos serviços de saúde e aos métodos contracetivos é desigual e existe baixa literacia em saúde sexual e reprodutiva na população portuguesa (Presado, Palma, \& Cardoso, 2018). Apesar da acessibilidade à informação acerca dos métodos contracetivos, continuamos a verificar um número significativo de gravidezes não planeadas e não desejadas relacionadas com a não adesão, descontinuação e uso incorreto dos métodos contracetivos e, necessidades insatisfeitas de planeamento familiar (Presado, Palma, \& Cardoso, 2018). 
No Relatório dos Registos das Interrupções Voluntárias de Gravidez de 2019, o número total de IVG foi de 14 306, correspondendo a 95,8\% do total de abortos realizados em Portugal, verificando-se uma tendência decrescente desde 2011. A faixa etária mais prevalente situase entre os $25-29$ anos (22,6\%) e os $20-24$ anos com (22.4\%), situando-se a média de idades nos 29,03 anos, demonstrando o aumento da idade média das mulheres que realizam uma IVG (DGS, 2019).

Os contracetivos hormonais oral e injetáveis são os métodos utilizados por $37,7 \%$ das mulheres (DGS, 2019) e também os mais conhecidos em Portugal (Bayer, 2017). No entanto, são os que apresentam maiores problemas de irregularidade, descontinuação e falhas na sua utilização (Bayer, 2017). Das mulheres utilizadoras de pilula, 84\% reconhecem já se ter esquecido da toma e $47 \%$ têm a intenção de mudar de método para a contraceção reversível de longa duração (Bayer, 2017).

Os anticoncecionais reversíveis de longa duração (LARC, do inglês Long-Acting Reversible Contraceptives e que incluem o sistema intrauterino (SIU), o dispositivo intrauterino (DIU) e o implante subcutâneo) são considerados os contracetivos com maior eficácia na redução de incidência de gravidezes não planeadas, apresentam menos efeitos secundários e aumentam a satisfação e a continuidade das utilizadoras (Manicholas, Madden, Secura, \& Peirent, 2014; Ricciotti et al., 2015), embora não sejam os métodos contracetivos mais utilizados $(37,4 \%)$ em Portugal (DGS, 2019).

Quando as barreiras dos custos, acesso e conhecimento são ultrapassadas, as mulheres escolhem os métodos mais eficazes e menos dependentes do utilizador, como é o caso dos LARC. Não só os escolhem como continuam a usá-los de forma satisfatória, evitando a gravidez não intencional (McNicholas, Tessa, Secura, \& Peipert, 2014).

Incentivar a utilização de LARC pode implicar a redução das taxas de gravidez indesejada e IVG, traduzindo-se em melhores condições económicas e financeiras, educacionais e sociais para as mulheres e famílias (McNicholas, Tessa, Secura, \& Peipert, 2014).

A vivencia de uma IVG promove a consciência do retorno iminente da fertilidade e a preocupação em conhecer as alternativas contracetivas eficazes, sendo, por isso, um momento oportuno para aconselhamento contracetivo. No período pós-IVG, a taxa de recetividade, adesão, continuidade e satisfação do método é alta, uma vez que aumenta a possibilidade da sua continuidade (Schunmann et al., 2016; Temmerman, 2019). 
Os profissionais de saúde têm um papel decisivo na educação para a saúde em planeamento familiar depois de uma IVG (Pereia, Palma, Godinho, \& Pereira, 2018), promovendo a capacitação das mulheres e companheiros na escolha informada e consciente do método contracetivo adequada às suas necessidades, expectativas e opções de vida.

O objetivo desta Revisão Sistemática é conhecer o impacto do aconselhamento contracetivo na escolha dos contracetivos pelas mulheres em processo de interrupção voluntária de gravidez.

\section{MÉTODO}

Foi realizada uma revisão sistemática da literatura segundo as recomendações do Joanna Briggs Institute (JBI) Reviewer's Manual, de 1 a 31 de janeiro de 2020, com o objetivo de reunir a evidência disponível, conforme os critérios de elegibilidade pré-especificados e para responder a uma questão específica: "Qual o aconselhamento contracetivo realizado às mulheres em processo de Interrupção Voluntária de Gravidez e qual o seu impacto na escolha do método?" considerou-se na pesquisa: $P$ (Patients) = Mulheres em processo de Interrupção Voluntária Gravidez; I (Intervention) = Aconselhamento sobre métodos contracetivos; C (Comparison) = Entre "Aconselhamento" Versus "Não-aconselhamento"; O (Outcome) $=$ Taxa de utilização dos Contracetivos e adesão aos Contracetivos.

As etapas percorridas nesta revisão sistemática foram: definição da questão de pesquisa e dos critérios de inclusão, pesquisa e seleção dos estudos, recolha de dados, avaliação de qualidade metodológica dos estudos incluídos, análise dos dados, identificação de vieses, sumarização e apresentação dos resultados, a sua interpretação e apresentação das conclusões.

Assim, definiram-se como critérios de inclusão dos estudos: a) todos os tipos de desenho de estudo; b) relato de estratégias dirigidas a mulheres com idade igual ou superior a 16 anos; c) sobre estratégias aplicadas por profissionais de saúde; d) sobre aconselhamento contracetivo durante o processo de IVG; e) sobre métodos de planeamento familiar prestado por hospitais, clínicas ou nos cuidados na comunidade; f) sem limite temporal; g) em português, inglês ou espanhol. 
E como critérios de exclusão dos estudos: a) conteúdo relativo a estratégias dirigidas a mulheres com idade inferior a 16 anos; b) conteúdo relativo a aborto por motivos médicos (de causa materna ou fetal); c) conteúdo relativo a estratégias dirigidas a mulheres que tenham sido vítimas de abuso sexual.

A estratégia de pesquisa foi desenvolvida segundo as três etapas definidas pelo JBI para a revisão sistemática da literatura: a) pesquisa inicial em bases de dados relevantes para o tema; b) pesquisa com palavras-chave e termos indexados nas bases de dados selecionadas; c) análise das referencias dos artigos selecionados para seleção de fontes adicionais.

A pesquisa inicial foi realizada na plataforma ESBCO, nas bases de dados CINAHL, MedLine, MedicLatina e Cochrane Central Register of Controlled Trials. A pesquisa realizouse através da análise das palavras-chave contidas nos títulos, resumos e nos termos indexados usados para descrever os artigos.

De acordo com o objetivo, foram utilizados os seguintes descritores: $\mathrm{MH}$ "Women"; $\mathrm{MH}$ "Contraceptive Devices"; MH "Contraceptive Agents"; MH "Family Planning Services"; MH "Abortion Induced"; "Post Abortion Contraception"; "Family Planning Counseling"; "Contraceptive Counseling". Realizou-se uma segunda pesquisa com todos os termos da questão (naturais e indexados) nas bases de dados referidas. Na última fase utilizaram-se os termos encontrados e os operadores booleanos "OR" e "AND". A estratégia de pesquisa na base de dados foi a seguinte: (MH "Contraceptive Devices" OR MH "Contraceptive Agents" OR MH "Family Planning Services" OR "Post Abortion Contraception") AND (MH "Women") AND (MH "Abortion Induced") AND ("Family Planning Counseling" OR "Contraceptive Counseling").

$\mathrm{Na}$ terceira etapa pesquisámos e analisámos as referências bibliográficas dos artigos elegíveis com o objetivo de identificar estudos adicionais. Os autores avaliaram a qualidade metodológica dos estudos utilizando os instrumentos preconizados pelo JBI (2015), nomeadamente: "Meta-Analysis of Statistics Assessment and Review Instrument (MAStARI) critical appraisal tools Comparable Cohort/Case Control Studies" e "MAStARI critical appraisal tools Descriptive/Case Series Studies". Foi também, utilizado o "MAStARI critical appraisal tools Randomized Control/ Pseudo-randomized Trial". Incluíram-se apenas os estudos que apresentaram um elevado nível de qualidade, score superior ou igual a 7. 


\section{RESULTADOS}

No processo de seleção dos estudos emergiram 69 artigos dos quais, foram excluídos 6 por se encontrarem em duplicado, 34 após leitura do título e resumo e 18 artigos por não corresponderem aos critérios de inclusão. Consideraram-se elegíveis 11 artigos para análise, após avaliação dos critérios de inclusão e exclusão descritos. Os títulos e resumos identificados a partir da pesquisa foram revistos de forma independente pelos revisores.

Após a seleção dos artigos, foi realizado um registo continuado e coordenado pelos autores com as principais informações dos estudos/artigos encontrados. A síntese dos dados relevantes recolhidos de cada artigo, considerando as orientações do $\mathrm{JB} /$ foi composta pelos seguintes itens: identificação do artigo; objetivo do estudo; caracterização da intervenção de aconselhamento; caracterização da população alvo e resultados da intervenção. Os onze artigos são estudos primários, desenvolvidos entre os anos 2014 e 2020. Todos na língua inglesa e realizados nos Estados Unidos (quatro), continente Asiático (três), Africano (dois), Europeu (um) e na Austrália (um). O tamanho das amostras variou entre 29 e 319385 mulheres. Os estudos incluídos apresentaram um elevado nível de qualidade (Tabela 1), score superior ou igual a 7 (seis com score 10, dois com 9, um com 8 e dois com 7).

Tabela 1. Identificação dos estudos selecionados.

\begin{tabular}{|c|c|c|c|c|c|}
\hline $\begin{array}{l}\text { Autores, } \\
\text { ano de } \\
\text { publicação } \\
\text { e área } \\
\text { geográfica }\end{array}$ & Objetivo & $\begin{array}{l}\text { Caracterização da } \\
\text { Intervenção }\end{array}$ & $\begin{array}{l}\text { Caracterização da } \\
\text { População Alvo da } \\
\text { Intervenção }\end{array}$ & Resultados & JBI \\
\hline $\begin{array}{l}\text { Madden et } \\
\text { al. } 2019, \\
\text { USA }\end{array}$ & $\begin{array}{l}\text { Comparar a taxa de } \\
\text { gravidez indesejada } \\
\text { aos } 12 \text { meses entre } \\
\text { mulheres que } \\
\text { recebem } \\
\text { aconselhamento } \\
\text { contracetivo padrão vs } \\
\text { completo (LARC). }\end{array}$ & $\begin{array}{l}\text { Serviço de } \\
\text { aconselhamento } \\
\text { contracetivo } \\
\text { realizado a dois } \\
\text { grupos diferentes de } \\
\text { mulheres. }\end{array}$ & $\begin{array}{l}502 \text { mulheres receberam } \\
\text { aconselhamento padrão; } \\
506 \text { mulheres receberam } \\
\text { aconselhamento completo } \\
\text { e suporte de custos aos } \\
\text { métodos LARC. }\end{array}$ & $\begin{array}{l}\text { Taxa de gravidez indesejada aos } 12 \\
\text { meses: } 5.3 \text { (Completo) vs } 9.8 \\
\text { (Padrão) gravidezes indesejadas em } \\
\text { cada 100. Mulheres que tiveram } \\
\text { aconselhamento completo } \\
\text { apresentaram um risco } 40 \% \text { inferior } \\
\text { de gravidez indesejada. }\end{array}$ & 10 \\
\hline $\begin{array}{l}\text { Tomnay et } \\
\text { al. } 2018, \\
\text { Australia }\end{array}$ & $\begin{array}{l}\text { Descrever a } \\
\text { população e os } \\
\text { resultados da } \\
\text { intervenção de uma } \\
\text { clínica de IVG- } \\
\text { medicamentosa em } \\
\text { ambiente rural. }\end{array}$ & $\begin{array}{l}\text { Serviço na } \\
\text { comunidade, } \\
\text { liderado por } \\
\text { enfermeira e com } \\
\text { apoio de médico de } \\
\text { família; Discussão } \\
\text { sobre métodos } \\
\text { contracetivos e } \\
\text { opções sobre a } \\
\text { gravidez. }\end{array}$ & $\begin{array}{l}\text { Método contracetivo antes } \\
\text { IVG: } 143(73.3 \%) \text { - } \\
\text { nenhum; } 3(1.5 \%) \text { pílula } \\
\text { do dia seguinte; } 10(2.1 \%) \\
\text { - preservativo; } 34(17.4 \%) \\
\text { - pílula; } 2(1.03 \%) \text { - } \\
\text { injetável; } 3(1.57 \%) \text { - } \\
\text { implante. }\end{array}$ & $\begin{array}{l}\text { Método contracetivo pós-IVG: } 21 \\
(10.8 \%) \text { - nenhum; } 66(33.8 \%)- \\
\text { pílula, } 27(13.8 \%) \text { - implante; } 18 \\
(9.2 \%) \text { - DIU; } 17-(8.7 \%) \text { injetável; } 3 \\
(1.5 \%) \text { - anel vaginal; } 8(4.1 \%)- \\
\text { vasectomia do parceiro; } 7(3.6 \%) \text { - } \\
\text { preservativo; } 1 \text { (0.5\%) - métodos } \\
\text { naturais. }\end{array}$ & 10 \\
\hline $\begin{array}{l}\text { Benson et } \\
\text { al. } 2018, \\
\text { Africa/Asia }\end{array}$ & $\begin{array}{l}\text { Descrever as } \\
\text { intervenções de } \\
\text { melhoria de } \\
\text { aconselhamento } \\
\text { contracetivo em } \\
\text { instituições de saúde. }\end{array}$ & $\begin{array}{l}\text { Desenvolvimento de } \\
\text { protocolos e guias; } \\
\text { Melhoria das } \\
\text { instituições; Treino } \\
\text { dos profissionais de } \\
\text { saúde; }\end{array}$ & $\begin{array}{l}\text { A IVG realizada no } \\
\text { primeiro trimestre }(\leq 12 \\
\text { semanas). } \\
\text { Mulheres com idade }>25 \\
\text { anos. }\end{array}$ & $\begin{array}{l}\text { Adesão a métodos contracetivos: } \\
73 \% \text {. } \\
\text { Método contracetivo pós gravidez: } \\
9 \% \text { - DIU; } 9 \% \text { - implante; } 30 \% \text { - } \\
\text { injetável; } 29 \% \text { - contracetivos orais; } \\
14 \% \text { - preservativo. }\end{array}$ & 10 \\
\hline
\end{tabular}




\begin{tabular}{|c|c|c|c|c|c|}
\hline $\begin{array}{l}\text { Wendot et } \\
\text { al. } 2018, \\
\text { Quénia }\end{array}$ & $\begin{array}{l}\text { Avaliar se o } \\
\text { planeamento familiar } \\
\text { pós-IVG e os LARC } \\
\text { aumentaram após } \\
\text { introdução de um } \\
\text { sistema de qualidade } \\
\text { em clínicas privadas. }\end{array}$ & $\begin{array}{l}\text { Intervenções } \\
\text { dirigidas a mulheres } \\
\text { que procuram } \\
\text { clínicas privadas } \\
\text { para a realização de } \\
\text { IVG. }\end{array}$ & $\begin{array}{l}883(53 \%) \text { mulheres foram } \\
\text { abrangidas pela } \\
\text { intervenção. } \\
\text { Método contracetivo antes } \\
\text { da gravidez: } 6.4 \% \text { - } \\
\text { nenhum; } 16.2 \% \text { - LARC } \\
16.2 \% ; 76.4 \% \\
\text { contracetivos de curta } \\
\text { duração; }\end{array}$ & $\begin{array}{l}\text { Não foram identificadas diferenças } \\
\text { significativas na adesão a métodos } \\
\text { contracetivos entre antes e após IVG. }\end{array}$ & 7 \\
\hline $\begin{array}{l}\text { Stembile et } \\
\text { al. } 2016, \\
\text { Africa } \\
\text { Ocidental }\end{array}$ & $\begin{array}{l}\text { Descrever estratégias } \\
\text { de melhoria no } \\
\text { aconselhamento } \\
\text { contracetivo e avaliar } \\
\text { a sua eficácia e uso } \\
\text { nas instituições de } \\
\text { saúde. }\end{array}$ & $\begin{array}{l}\text { Treino dos } \\
\text { profissionais de } \\
\text { saúde em } \\
\text { programas de } \\
\text { aconselhamento } \\
\text { contracetivo; } \\
\text { estratégias de } \\
\text { aconselhamento } \\
\text { após-IVG (LARC). }\end{array}$ & $\begin{array}{l}749(91 \%) \text { das mulheres } \\
\text { foram abrangidas pela } \\
\text { intervenção de } \\
\text { aconselhamento. } \\
\text { Método contracetivo antes } \\
\text { IVG: } 81 \% \text { - pílula; } 4 \% \text { - } \\
\text { implante; } 4 \% \text { - DIU. }\end{array}$ & $\begin{array}{l}\text { Adesão aos métodos contracetivos } \\
\text { aumentou de } 37 \% \text { para } 60 \% \text { na } \\
\text { população do estudo. } \\
\text { Métodos contracetivos pós-IVG: } 32 \% \\
\text { - pílula; } 27 \% \text { - implante. Apenas um } \\
\text { centro participante tinha DIU; } 9 \% \text { das } \\
\text { mulheres nesse centro adotaram este } \\
\text { método. }\end{array}$ & 10 \\
\hline $\begin{array}{l}\text { Merki-Feld } \\
\text { et al. } 2018, \\
\text { Europe. }\end{array}$ & $\begin{array}{l}\text { Avaliar os cuidados } \\
\text { dos prestadores de } \\
\text { saúde em torno da } \\
\text { contraceção e do uso } \\
\text { de métodos } \\
\text { contracetivos. }\end{array}$ & $\begin{array}{l}\text { Descrever através } \\
\text { de um questionário } \\
\text { a utilização dos } \\
\text { métodos } \\
\text { contracetivos, } \\
\text { satisfação e método } \\
\text { utilizado. }\end{array}$ & $\begin{array}{l}\text { Foram realizados } \\
\text { questionários online a } 676 \\
\text { profissionais de saúde e } \\
6027 \text { mulheres. }\end{array}$ & $\begin{array}{l}\text { Utilização de um método } \\
\text { contracetivo: } 90 \% \text {. } \\
55 \% \text { - método de curta duração; } 18 \% \\
\text { - LARC. A utilização dos LARC varia } \\
\text { de } 7 \% \text { a } 38 \% \text { (Portugal vs França). } \\
73 \% \text { das mulheres considerariam os } \\
\text { LARC. }\end{array}$ & 8 \\
\hline $\begin{array}{l}\text { Pearson et } \\
\text { al. } 2017, \\
\text { Bangladesh }\end{array}$ & $\begin{array}{l}\text { Descrever as taxas de } \\
\text { adesão e contraceção } \\
\text { após aborto e quais } \\
\text { os fatores que } \\
\text { condicionam a adesão } \\
\text { aos contracetivos. }\end{array}$ & $\begin{array}{l}\text { Acompanhamento } \\
\text { clínico das mulheres } \\
4 \text { meses após IVG. }\end{array}$ & $\begin{array}{l}\text { Mulheres que sofreram } \\
\text { aborto espontâneo ou IVG } \\
\text { pelo método } \\
\text { medicamentoso ou } \\
\text { cirúrgico. }\end{array}$ & $\begin{array}{l}\text { Contraceção pós-IVG: } 76.2 \% \text {. } \\
\text { Contraceção } 4 \text { meses pós-IVG: } \\
85.4 \% \text {. } \\
\text { Aconselhamento deve ser } \\
\text { confidencial e especializado. }\end{array}$ & 9 \\
\hline $\begin{array}{l}\text { Che et al. } \\
2017 \text {, China }\end{array}$ & $\begin{array}{l}\text { Descrever estratégias } \\
\text { de melhoria do serviço } \\
\text { de planeamento } \\
\text { familiar. }\end{array}$ & $\begin{array}{l}\text { Avaliar a eficácia de } \\
\text { integrar } \\
\text { aconselhamento } \\
\text { familiar pós-IVG. } \\
\text { Consulta presencial, } \\
\text { realizada por casal. }\end{array}$ & $\begin{array}{l}\text { Mulheres que que } \\
\text { realizaram IVG nos } \\
\text { últimos } 6 \text { meses antes da } \\
\text { entrevista. }\end{array}$ & $\begin{array}{l}\text { Adesão aos métodos contracetivos } \\
\text { está relacionada com a informação } \\
\text { transmitida nas consultas de } \\
\text { aconselhamento. }\end{array}$ & 10 \\
\hline $\begin{array}{l}\text { Laursen et } \\
\text { al. } 2017 \text {, } \\
\text { USA }\end{array}$ & $\begin{array}{l}\text { Comparar a } \\
\text { contraceção oferecida } \\
\text { às mulheres após IVG } \\
\text { medicamentosa e } \\
\text { cirúrgica; adesão aos } \\
\text { métodos } \\
\text { contracetivos. }\end{array}$ & $\begin{array}{l}\text { Determinar o tipo de } \\
\text { intervenção a que } \\
\text { as mulheres são } \\
\text { sujeitas. Método } \\
\text { contracetivo } \\
\text { escolhido após IVG } \\
\text { medicamentosa ou } \\
\text { cirúrgica. }\end{array}$ & $\begin{array}{l}\text { Mulheres que realizaram } \\
\text { IVG medicamentosa ou } \\
\text { cirúrgica. }\end{array}$ & $\begin{array}{l}\text { IVG medicamentosa: } 237 \text { (28.8\%) } \\
\text { mulheres } \\
\text { IVG cirúrgica: } 587 \text { (71.2\%) mulheres } \\
\text { Taxa de adesão aos LARC: } 41.9 \% \\
\text { (IVG cirúrgica) vs } 23.2 \% \text { (IVG } \\
\text { medicamentosa). } \\
\text { Taxa de adesão a um método } \\
\text { contracetivo: } 83 \% \text { (IVG cirúrgica) vs } \\
64.6 \% \text { (IVG medicamentosa). }\end{array}$ & 9 \\
\hline $\begin{array}{l}\text { Tang et al. } \\
2017 \text {, China }\end{array}$ & $\begin{array}{l}\text { Avaliar a prática de } \\
\text { aconselhamento no } \\
\text { planeamento familiar } \\
\text { após-IVG entre os } \\
\text { profissionais de } \\
\text { saúde. }\end{array}$ & $\begin{array}{l}\text { Aconselhamento } \\
\text { contracetivo } \\
\text { realizado pelos } \\
\text { profissionais de } \\
\text { saúde. }\end{array}$ & $\begin{array}{l}\text { Todos os profissionais de } \\
\text { saúde que em instituições } \\
\text { com serviços de IVG } \\
\text { foram convidados a } \\
\text { participar num } \\
\text { questionário. }\end{array}$ & $\begin{array}{l}\text { 92\% dos participantes reportaram } \\
\text { promover o aconselhamento } \\
\text { contracetivo; } 57 \% \text { despendem mais } \\
\text { de } 10 \text { minutos para esse efeito. } \\
\text { A formação dos profissionais de } \\
\text { saúde esteve correlacionada com a } \\
\text { modalidade de aconselhamento } \\
\text { prestado. }\end{array}$ & 10 \\
\hline $\begin{array}{l}\text { Rocca et al. } \\
2018 \text {, USA }\end{array}$ & $\begin{array}{l}\text { Descrever as } \\
\text { diferenças entre } \\
\text { aconselhamento } \\
\text { contracetivo e escolha } \\
\text { de métodos } \\
\text { contracetivos entre } \\
\text { IVG medicamentosa e } \\
\text { cirúrgica }\end{array}$ & $\begin{array}{l}\text { Escolha e uso de } \\
\text { métodos } \\
\text { contracetivos; } \\
\text { Treino dos } \\
\text { profissionais nos } \\
\text { métodos LARC. }\end{array}$ & $\begin{array}{l}\text { Mulheres entre os } 18-25 \\
\text { anos que não queriam } \\
\text { engravidar foram } \\
\text { acompanhadas durante } 1 \\
\text { ano. }\end{array}$ & $\begin{array}{l}\text { IVG medicamentosa: } 166 \text { (26\%) } \\
\text { mulheres } \\
\text { IVG cirúrgica: } 477 \text { ( } 74 \%) \text { mulheres } \\
\text { Mulheres que optaram pela IVG } \\
\text { cirúrgica escolheram } \\
\text { preferencialmente o DIU no primeiro } \\
\text { ano. }\end{array}$ & 7 \\
\hline
\end{tabular}

Dos artigos elegíveis para análise verificou-se que os estudos pretenderam: descrever as caraterísticas da população (Tomnay et al., 2018); os resultados de intervenções em 
aconselhamento contracetivo no pós-aborto (Benson et al., 2018; Tomnay et al., 2018; Wendot et al., 2018); compará-los com as taxas de gravidezes não desejadas (Madden et al., 2019); as estratégias para o melhor aconselhamento contracetivo (Stembile, Kassouta, Sebikali, Lundstrom, \& Saade, 2016; Che et al., 2017); aconselhamento realizado pelos profissionais de saúde em torno da contraceção e dos métodos contracetivos (Merki-Feld, Caetano, Porz, \& Bitzer, 2018); a sua avaliação no pós-IVG (Tang et al., 2017); descrever a taxa de adesão à contraceção após aborto e os fatores que condicionam essa adesão (Pearson et al., 2017); relação entre o fornecimento de contracetivos após a IVG e a sua adesão (Laursen, Stumbras, Lewnard, Haider, \& Laursen, 2017); diferenças no aconselhamento contracetivo e as escolhas de um método no pós-IVG (Rocca et al., 2018).

\section{DISCUSSÃO}

A evidência diz-nos que o perfil contracetivo das mulheres antes de realizarem a IVG é de que, a grande maioria não faz contraceção, seguido das que utilizam a pilula contracetiva e com percentagem residual as que recorrem à contraceção de emergência, preservativo, hormonal injetável e implante (Tomnay et al., 2018). Os estudos, dizem-nos que a maioria das mulheres escolhe um contracetivo após a IVG (Tomnay et al., 2018; Wendot et al., 2018; Merki-Feld, Caetano, Porz, \& Bitzer, 2018).

Numa amostra de 152 mulheres, 21 (10,8\%) não iniciaram qualquer tipo de contraceção após a IVG, a 66 (33,8\%) optaram pela pilula, 27 (13,8\%) pelo implante, 18 (9,2\%) pelo DIU, $17(8,7 \%)$ por o hormonal injetável, $3(1,5 \%)$ escolheram o anel contracetivo, $8(4,1 \%)$ realizaram vasectomia, $7(3,6 \%)$ usam o preservativo e $1(0,5 \%)$ os métodos naturais (Tomnay et al., 2018).

Outros estudos corroboram que $73 \%$ das mulheres adotaram um contracetivo após a IVG, dividindo-se entre o hormonal injetável (30\%), pilula (29\%), preservativo (14\%) e um número residual escolheu o DIU (9\%) (Benson et al., 2018). Wendot et al. (2018) acrescentam que $6.4 \%$ não faz contraceção após a IVG, 76\% escolher a pilula contracetiva e $16.2 \%$ optam por métodos mais eficazes como os $L A R C$.

Verifica-se que a proporção de escolha e adesão aos DIUs aumenta com a idade das mulheres (Tomnay et al., 2018) e estado civil (casadas) (Che et al., 2017), sendo baixa nas mulheres com idades inferiores aos 25 anos (Benson et al., 2018). Também se verificou que existem diferenças na escolha dos $\angle A R C$ entre os diferentes países variando a sua 
utilização entre 7\% em Portugal e 38\% em França (Merki-Feld, Caetano, Porz, \& Bitzer, 2018).

Reconhece-se que o aconselhamento contracetivo leva ao aumento da adesão aos métodos contracetivos de $37 \%$ para $60 \%$, sendo os mais selecionados as pilulas (32\%) e o implante (27\%). O DIU foi selecionado por $9 \%$ das mulheres podendo essa percentagem estar associada ao fato de nem todos os centros de aconselhamento possuírem este contracetivo (Stembile, Kassouta, Sebikali, Lundstrom, \& Saade, 2016). A evidência mostra-nos que o aconselhamento contracetivo deve ser centrado nas necessidades da mulher em processo de IVG (Pearson et al., 2017) e que a adesão aos métodos contracetivos está relacionada com a qualidade da informação transmitida nas consultas da aconselhamento (Che et al., 2017). O aconselhamento contracetivo sobre métodos eficazes e modernos como os LARC (Madden et al., 2019), a acessibilidade às consultas de planeamento familiar, a educação para a saúde por profissionais treinados (Che et al., 2017) e o suporte dos custos dos contracetivos foram estratégias capazes de reduzir a probabilidade das mulheres ficarem expostas a uma gravidez não planeada em até 40\% (Madden et al., 2019). A maioria das mulheres $(60 \%)$ mostrou interesse em receber mais informação sobre contraceção, sendo que $73 \%$ admitiu considerar a aplicação de um LARC (Merki-Feld, Caetano, Porz, \& Bitzer, 2018) e 76.2\% adotou um contracetivo após a IVG (Pearson et al., 2017).

As mulheres que realizaram uma IVG cirúrgica mostraram maior adesão à contraceção do que as que realizaram uma IVG pelo método medicamentoso (83\% versus $64.6 \%$ ). O mesmo acontece com a escolha de um LARC (41.9\% versus 23.3\%) (Laursen et al., 2017). Não obstante acredita-se que esse facto se deveu à acessibilidade aos serviços de saúde (Rocca et al., 2018).

Os profissionais de saúde que integram as equipas de IVG mostram-se recetivos em promover aconselhamento contracetivo (92\%), mas apenas $57 \%$ despendem mais de dez minutos para o efeito.

Os profissionais que detêm mais formação despendem mais tempo no aconselhamento, influenciando a qualidade do aconselhamento prestado (Tang et al., 2017).

Os autores, defendem que a formação dos profissionais de saúde em planeamento familiar como parte integrante do processo de IVG são essenciais para fornecer aconselhamento contracetivo de qualidade. 


\section{CONCLUSÕES}

$\mathrm{Na}$ literatura disponível sobre aconselhamento em contraceção no pós-aborto, foram identificados onze estudos publicados entre 2014 e 2020, publicados nos Estados Unidos (quatro), continente Asiático (três), Africano (dois), Europeu (um) e na Oceânia (um). Não foram identificados artigos em português acerca do tema em estudo.

As orientações em aconselhamento contracetivo tendem a ser pouco objetivas do ponto de vista social/comportamental. A Organização Mundial de Saúde (2018) tem orientações focadas nos critérios clínicos do aconselhamento, mas carecem de orientações relativas às dimensões sociais e psicológicas das mulheres.

$\mathrm{Na}$ evidência encontrada, demarcámos as principais conclusões em dois grandes grupos: o aconselhamento e a escolha e adesão a um contracetivo. Relativamente ao aconselhamento, sobressai que: o aconselhamento favorece a adesão aos métodos contracetivos em geral; o aconselhamento favorece a prescrição dos $L A R C$ em particular; nem sempre é feito aconselhamento e quando existe é muito heterogéneo; os recursos disponíveis influenciam a qualidade de resposta; muitas vezes não é adaptado à realidade individual; a qualidade do aconselhamento depende da qualificação do profissional e o aconselhamento deve ser centrado nas necessidades das mulheres.

$\mathrm{Na}$ escolha e adesão de/aos contracetivos: o financiamento dos contracetivos favorece a sua escolha e a sua adesão; a acessibilidade aos serviços de saúde influencia a escolha dos métodos; a IVG pelo método cirúrgico favorece a adesão à contraceção e, em especial, aos $L A R C$; o momento de introdução de um contracetivo influencia a adesão ao mesmo e o pós-IVG é o momento ideal para aderir a um contracetivo.

Em síntese podemos inferir que os $L A R C$ s podem ser considerados a escolha de primeira linha das mulheres (independentemente da idade), dada a evidência demonstrada na sua satisfação, continuidade, benefícios económicos e superioridade em termos de efetividade face aos outros contracetivos.

O período da experiência vivida de uma IVG parece ser um momento ideal para o aconselhamento, orientação e escolha do método contracetivo e deve ocorrer desde o primeiro contacto da mulher aos serviços de saúde. 
Os profissionais de saúde, têm um papel preponderante na educação para a saúde sexual e reprodutiva.

Reconhecemos as limitações desta revisão onde percebemos que os contextos epidemiológicos eram muitos distintos, com amostras muito variáveis, contextos clínicos distintos, diferenças culturais, sociais e a ausência de estudos portugueses ou de língua portuguesa (para estes critérios de elegibilidade).

Os aspetos psicossociais da mulher como a relação com o parceiro, a influência da violência doméstica, crenças culturais ou religiosas e a vivencia emocional são aspetos pouco abordados na literatura.

Da mesma forma, os profissionais de saúde, no exercício da sua prática estão sujeitos ao contexto psicossocial e encontram desafios que vão para além das orientações clínicas.

Os autores acreditam que a investigação qualitativa, na forma de relato verbal ou escrito, tanto das mulheres como dos profissionais, pode representar uma forma de avaliar 0 impacto dos aspetos psicossociais.

Além disso, os autores defendem que as linhas de investigação futuras devem analisar contextos epidemiológicos de interesse (Portugal), com maior número de IVG, no intuito de serem desenvolvidas intervenções no campo do aconselhamento contracetivo centrados na pessoa e que possam servir de base para boas práticas de cuidados a estas mulheres.

Financiamento: A Bayer apoiou o desenvolvimento deste manuscrito através do financiamento do medical writing, fornecido por um parceiro externo.

Conflitos de Interesse: As autoras Sara Palma, Nídia Nunes e Adriana Taborda declaram ter recebido honorários como palestrantes para a Bayer.

Agradecimentos: À ESEL a possibilidade de aceder à plataforma ESBCO sem a qual esta revisão não seria possível e à Bayer o desafio lançado e o interesse pelo estudo cientifico em contraceção.

\section{REFERÊNCIAS}

Águas, F., Bombas, T. \& Silva, D. P. (2016). Avaliação das práticas contracetivas das mulheres em Portugal. Acta Obstet Ginecol Port. 10(3), 184-192. Retrieved from: http://www.scielo.mec.pt/pdf/aogp/v10n3/v10n3a02.pdf

Bayer (2017). Pílula contracetiva: o método mais utilizado mas também muito esquecido. Geração milénio e contraceção: Porque nos esquecemos? Raio $X$ Jornal de Saúde On-line. Retrieved from: http://raiox.pt/pilula-contracetiva-metodo-utilizado-tambem-esquecido/ 
Benson, J., Andersen, K., Brahmi, D., Healy, J., Mark, A., Ajode, A. \& Griffin, R. (2018). "What contraception do women use after abortion? An analysis of 319,385 cases from eight countries". Global Public Health. Retrieved from: https://www.tandfonline.com/doi/pdf/10.1080/17441692.2016.1174280

Che, Y., Dusabe-Richards, E., Wu, S., Jiang, Y., Dong, X., Li, J., Zhang, W. H., ... Tolhurst, R.(2017). "A qualitative exploration of perceptions and experiences of contraceptive use, abortion and post-abortion family planning services (PAFP) in three provinces in China". BMC Women's Health, 17(1), 113.

Direção-Geral da Saúde. (2019). Relatório dos registos das interrupções da gravidez: 2018. Retrieved from: https://www.dgs.pt/portal-da-estatistica-da-saude/diretorio-de-informacao/diretorio-de-informacao/por-serie1144918-pdf.aspx?v=\%3D\%3DDwAAAB\%2BLCAAAAAAABAArySzItzVUy81MsTU1MDAFAHzFEfkPAAAA

Gomes, C. C., Fernanda, V., Almeida, D.C., Gonçalves, E., Viana, J., Pacheco, A. \& Silva, A.P. (2018). Contraceção e interrupção voluntária de gravidez: $O$ antes e o depois. $8^{a}$ Reunião Nacional da Sociedade Portuguesa de Contraceção. Retrieved from: http://www.spdc.pt/images/livro_de_resumos.pdf

Greene, M., Joshi, S. \& Robles, O. (2012). UNFPA: By choice, not by chance: Family planning, human rights and development. New York (NY): United Nations Population Fund (UNFPA). Retrieved from: http://www.unfpa.org/webdav/site/global/shared/swp/2012/EN_SWOP2012_Report.pdf

JBI (2015). The Joanna Briggs Institute Reviwer's Manual 2015 - Methodology for JBI Reviwes. Acedido em: 12/02/2019. Disponível em: http://joannabriggs.org/assets/docs/sumari/ReviewersManual_Methodologyfor-JBI-Scoping-Reviews_2015_v2.pdf

Laursen, L., Stumbras, K., Lewnard, I., Haider \& S. Laursen, L. (2017). "Contraceptive Provision after Medication and Surgical Abortion". Women's Health Issues, 27(5), 546-550.

Lei 16/2007 de 17 abril (2007). Exclusão de ilicitude nos casos de interrupção voluntária de gravidez. Assembleia da República. Diário da Republica I Série (№ 75, 17/04/1997) 2417-2418. Retrieved from: Retrieved from: http://www.saudereprodutiva.dgs.pt/legislacao/ interrupcaovoluntariadagravidez/lei-n162007-publicado-a17-de-abril.aspx

Madden, T., Paul, R., Maddipati, R., Buckel, C., Goodman, M., \& Peipert, J. F. (2019). "Comparison of unintended pregnancy at 12 months between two contraceptive care programs; a controlled time-trend design". Contraception (CONTRACEPTION). 100(3), 196- 201.

McNicholas, C., Tessa, M., Secura, G., \& Peipert, J.F. (2014). The Contraceptive CHOICE Project Round Up: what we did and what we learned. Clin Obstet Gynecol., 57(4), 635-643. Retrieved from: https://www.ncbi.nlm.nih.gov/pmc/articles/PMC4216614/

Merki-Feld, G. S., Caetano, C., Porz T.C., \& Bitzer J. (2018). Are there unmet needs in contraceptive counselling and choice? Findings of the European TANCO Study. Eur J Contracept Reprod Health Care, 23(3), 183193. doi: 10.1080/13625187.2018.1465546. Epub 2018 May 22.

Organização Mundial de Saúde. (2018). Guia de Implementação dos Critérios Médicos de Elegibilidade e das Recomendações da OMS para 0 Uso de Contraceção. Retrieved from: https://apps.who.int/iris/bitstream/handle/10665/272758/9789247513573-por.pdf?ua=1

Palma, S., \& Presado, H. (2019). Motivos que levam as mulheres a optarem por uma interrupção voluntária da gravidez: uma Scoping Review. Pensar em Enfermagem, 23(1).

Pearson, E., Andersen, K. L., Moreau, C., Chowdhury, R., Sultana, S., Shahidulla, H. S. M., ... Decker, M. R. (2017). Correlates of contraceptive use 4 months postabortion: findings from a prospective study in Bangladesh. Contraception, 95(3), 279-287.

Pereira, J. P., Palma, S., Godinho, B., \& Pereira, A. (2018). Contraceção Antes E Após Uma Interrupção Voluntária de Gravidez. Contraceção e interrupção voluntária de gravidez: $O$ antes e o depois. $8^{a}$ Reunião Nacional da Sociedade Portuguesa de Contraceção. Retrieved from: 
http://www.spdc.pt/images/livro_de_resumos.pdf

Presado, M. H., Palma, S., \& Cardoso, M. (2018). Vivências de um grupo de mulheres portuguesas em processo de interrupção voluntária da gravidez. Investigação Qualitativa em Saúde, ( vol. 2). In Proceedings por $7^{\circ}$ Congresso Ibero-Americano em Investigação Qualitativa, (pp. 414-422). Retrieved from: https://proceedings.ciaiq.org/index.php/ciaiq2018/article/view/1803/1756

Ricciotti, H. A., Dodge, L. E., Ramirez, C. I., Barnes, K., \& Hacker, M. R. (2015). Long-acting reversible contraceptive use in urban women from a title x-supported Boston community health center. J Prim Care Community Health, 6(2), 111-115. Retrieved from: https://doi.org/10.1177/2150131914553800

Rocca, C. H., Goodman, S., Grossman, D., Cadwallader, K., Thompson, K. M. J., ... Harper, C. C. (2018). Contraception after medication abortion in the United States: results from a cluster randomized trial. American Journal Of Obstetrics And Gynecology, 218(1), 1-107.

Schunmann, C., \& Glasier, A. (2016). Specialist contraceptive counselling and provision after termination of pregnancy improves uptake of long-acting methods but does not prevent repeat abortion: A randomized trial. Hum Reprod. 21(9), 2296-2303. Retrieved from: https://doi.org/10.1093/humrep/del168

Stembile, M., Kassouta, N., Sebikali, B., Lundstrom, L., \& Saade, A. (2016). Improving the Quality of Postabortion Care Services in Togo Increased Uptake of Contraception. Global Health: Science and Practice, 4(3), 495-505.

Tang, L., Wu, S., Li, J., Wang, K., Xu, J., Temmerman, M., \& Zhang, W. (2017). Post-abortion family planning counselling practice among abortion service providers in China: a nationwide cross-sectional study. European Journal of Contraception \& Reproductive Health Care, 22(1), 24-29.

Temmerman, M. (2019) Missed opportunities in women's health: post-abortion care. The Lancet Global Health. 7(1), e12-e13. Acedido a 08/02/2019. Disponível em: https://doi.org/10.1016/S2214-109X(18)30542-4

Tomnay, J. E., Coelli, L., Davidson, A., Hulme-Chambers, A., Orr, C., \& Hocking, S. (2018). Providing accessible medical abortion services in a Victorian rural community: A description and audit of service delivery and contraception follow up. Sexual \& Reproductive HealthCare, 16, 175-180.

Wendot, S., Rachel, H. S., Nafula, I., Theuri, I., Ikiugu, E., \& Footman, K. (2018). Evaluating the impact of a quality management intervention on post-abortion contraceptive uptake in private sector clinics in western Kenya: a pre- and post-intervention study. Reprod Health, 15(10) Retrieved from: https://doi.org/10.1186/s12978-018-0452-4 